\title{
Morbimortalidade por câncer infantojuvenil associada ao uso agrícola de agrotóxicos no Estado de Mato Grosso, Brasil
}

\author{
Morbidity and mortality from cancer children and adolescents \\ associated with the agricultural use of pesticides in the state of \\ Mato Grosso, Brazil
}

\author{
Hélen Rosane Meinke Curvo¹, Wanderlei Antônio Pignati², Marta Gislene Pignatti
}

\begin{abstract}
Resumo
O câncer infantojuvenil vem se apresentando como a segunda causa de óbito na população entre 0 e 19 anos no Brasil, atrás apenas dos acidentes. Dentre os fatores ambientais, para além das questões genéticas, estudos epidemiológicos relacionaram exposição a agrotóxicos e tumores da infância, especialmente leucemia. No interior do estado de Mato Grosso, o aumento do cultivo de grãos e exportação vem sendo acompanhado de uso crescente de agrotóxicos. Para analisar a associação entre o uso agrícola de agrotóxico e morbimortalidade por câncer em menores de 20 anos, foi realizado estudo ecológico da média das séries históricas de morbidade (2000-2005) e mortalidade (2000-2006) por câncer na faixa etária de 0 a 19 anos e o uso de agrotóxicos nos municípios do estado de Mato Grosso. Observou-se que a média de uso de agrotóxicos nos municípios apresentou associação estatisticamente significante tanto para morbidade $(p=0,021)$, como para mortalidade $(p=0,005)$ por câncer infantojuvenil, com intervalo de confiança de $95 \%$. Os resultados indicam que a exposição aos agrotóxicos está associada à morbimortalidade por câncer na população infantojuvenil. Recomenda-se como medida preventiva para evitar o câncer a redução do uso intencional dos agrotóxicos.
\end{abstract}

Palavras-chave: neoplasias; mortalidade; morbidade; praguicidas.

\begin{abstract}
Cancer in children and adolescents is reported as the second cause of death in the population between 0 and 19 years in Brazil, only behind the accidents. Among environmental factors, in addition to genetic issues, epidemiological studies have linked exposure to pesticides and childhood tumors, especially leukemia. Inside the state of Mato Grosso, the increase in grain cultivation and export has been accompanied by increasing use of pesticides. To analyze the association between the agricultural use of pesticides and cancer morbidity and mortality in children under 20 years, it was conducted ecological study of the average time series of morbidity (2000-2005) and mortality (2000-2006) from cancer at the age 0-19 years and the use of pesticides in municipalities in the state of Mato Grosso. It was observed that the average use of pesticides in the counties showed a statistically significant association for both morbidity $(p=0.021)$, as for mortality $(p=0.005)$ for cancer children and adolescents, with 95\% confidence interval. The results indicate that exposure to pesticides is associated with morbidity and mortality from cancer in children and adolescents. It is recommended as a preventive measure to prevent cancer to reduce the intentional use of pesticides.
\end{abstract}

Keywords: neoplasms; mortality; morbidity; pesticides.

Trabalho realizado no Instituto de Saúde Coletiva da Universidade Federal de Mato Grosso (UFMT) - Cuiabá (MT), Brasil.

'Mestre em Saúde Coletiva pelo Instituto de Saúde Coletiva da UFMT - Cuiabá (MT), Brasil.

2Professor Adjunto III do Instituto de Saúde Coletiva da UFMT - Cuiabá (MT), Brasil.

${ }^{3}$ Professora Associada II do Instituto de Saúde Coletiva da UFMT - Cuiabá (MT), Brasil.

Endereço para correspondência: Hélen Rosane Meinke Curvo - Avenida Rubens de Mendonça, 3.061/1.003 - Bosque da Saúde - CEP: 78050-000 - Cuiabá (MT),

Brasil-E-mail: helen.curvo@hotmail.com

Conflito de interesse: nada a declarar. 


\section{INTRODUÇÃO}

No Brasil, o câncer já representa a segunda causa de mortalidade proporcional entre crianças e adolescentes de 1 a 19 anos, para todas as regiões, assim como em países desenvolvidos. Tendo em vista que a primeira causa está relacionada aos acidentes e à violência, pode-se dizer que o câncer é a primeira causa de mortes por doença, após um ano de idade, até o final da adolescência ${ }^{1}$.

Dentre os fatores ambientais relacionados com este aumento para além das questões genéticas, Zahm e Ward ${ }^{2}$ publicaram extensa revisão de estudos epidemiológicos com associação entre exposição a agrotóxicos e tumores da infância, especialmente leucemia, tumores do sistema nervoso central (SNC) e linfoma não Hodgkin.

O câncer infantil corresponde a um grupo de várias doenças que têm em comum a proliferação descontrolada de células anormais e que pode ocorrer em qualquer local do organismo. Esse tipo de câncer varia de acordo com o tipo histológico, localização primária do tumor, etnia, sexo e idade. Merece lugar de destaque, pois representa, nessa faixa etária, importante causa de mortalidade e perda de potenciais anos de vida. Além disso, o desgaste psíquico, social e financeiro confere um impacto profundo nos pacientes, nas famílias, na sociedade e no sistema público de saúde $e^{3,4}$.

O câncer infantojuvenil é considerado raro quando comparado com os tumores do adulto, correspondendo entre 2 e $3 \%$ entre todos os tumores malignos e, geralmente, afeta as células do sistema sanguíneo e os tecidos de sustentação, enquanto que, o câncer do adulto afeta as células do epitélio, que recobre os diferentes órgãos (câncer de mama, câncer de pulmão). Apresenta ainda, tendência a menores períodos de latência, crescimento rápido e invasivo, porém responde melhor aos métodos terapêuticos atuais ${ }^{1,3-5}$.

Pelas diferenças de apresentação da doença nos locais primários, origens histológicas e comportamentos clínicos, o câncer nesta faixa etária deve ser estudado separadamente do câncer do adulto.

As neoplasias mais frequentes entre crianças e adolescentes são as leucemias, os tumores do sistema nervoso central e os linfomas. Mais raros, mas igualmente típicos são neuroblastoma, tumores renais (tumor de Willms), retinoblastoma, tumores germinativos, osteossarcomas e sarcomas de tecidos moles ${ }^{6}$.

Dos óbitos por neoplasias na infância, as leucemias representam a maior causa, sendo responsáveis por 39\% das mortes na Europa ${ }^{7}$ e por 50\% nas Américas, Oceania e Ásia ${ }^{8}$.

Os linfomas e neoplasias retículo-endoteliais correspondem ao terceiro tipo de câncer mais comum em crianças norte-americanas, após as leucemias e os tumores do SNC99. Já nos países em desenvolvimento correspondem ao segundo lugar, ficando atrás apenas das leucemias 5 .
Os linfomas não Hodgkin que ocorrem nas crianças correspondem a um grupo heterogêneo com diversos tipos histológicos, sendo mais comum o tipo Burkitt. Segundo informações do Surveillance, Epidemiology, and End Results (SEER), a incidência permanece constante na faixa etária entre 5 e 14 anos, porém, a incidência nos adolescentes de 15 a 19 anos passou de 10,7 por milhão, entre 1975-1979, para 16,3 por milhão no período $1990-1995^{10}$.

A avaliação do potencial carcinogênico dos agrotóxicos é muito complexa e envolve aspectos como a heterogeneidade dos compostos utilizados, as mudanças frequentes de produtos específicos, a variabilidade nos métodos de aplicação, a ausência de dados adequados sobre a natureza da exposição, o longo período necessário para a indução do câncer e as dificuldades na obtenção de dados sobre exposição ambiental ${ }^{11,12}$.

Sanborn et al..$^{13}$ publicaram revisão sistemática sobre o câncer infantojuvenil e o uso de agrotóxicos. Encontraram forte e consistente associação entre crianças que tiveram exposições mais longas e com maiores doses de agrotóxicos e linfoma não Hodgkin e leucemia. O estudo concluiu que seus resultados apoiam tentativas de reduzir a exposição a agrotóxicos como uma medida para evitar câncer.

Crianças e adolescentes que vivem nas áreas de cultivo ou próximas a elas podem estar expostas através da aplicação agrícola, da deriva das aplicações e/ou do excesso de pulverização. Crianças pequenas, que permanecem no chão ou solo e que colocam as mãos e objetos na boca, podem estar mais sujeitas à exposição. Além disso, os pais expostos de forma ocupacional podem trazer agrotóxicos para casa nas roupas e equipamentos, aumentando o risco de adoecimento por câncer dos filhos e o risco também aumenta de acordo com o grau de exposição das mães durante a gravidez ${ }^{2}$.

No interior do estado de Mato Grosso, com o crescimento da produção agrícola temporária (soja, algodão, milho, arroz, cana-de-açúcar, entre outros) nestes últimos 20 anos, houve incremento na utilização de agrotóxicos, com pulverização por avião e trator, próximo a fontes de água potável, córregos, criação de animais, residências, escolas e periferia dos municípios, o que pode estar refletindo no aumento de casos de câncer infantojuvenil ${ }^{14,15}$.

Com o objetivo de analisar a associação entre o câncer infantojuvenil e o uso agrícola de agrotóxicos no estado, este artigo foi delineado.

\section{MÉTODOS}

Para analisar a associação entre o uso agrícola de agrotóxicos nos municípios do estado em relação à morbidade e mortalidade 
por câncer em residentes menores de 20 anos - partindo-se do pressuposto de que esta população sofreu a exposição em sua área de residência - , foi realizado estudo epidemiológico descritivo com delineamento ecológico de séries históricas das informações sobre os indicadores de morbidade (2000-2005) e mortalidade (2000-2006) por câncer na faixa etária de 0 a 19 anos e o uso de agrotóxicos nos municípios do estado de Mato Grosso.

\section{Morbidade}

A incidência é conhecida pelos registros de câncer de base populacional (RCBP), que através de um processo contínuo e sistemático de coleta de dados registra todos os casos novos de câncer que ocorram em uma determinada população de uma área geográfica definida. Foram utilizados casos novos de neoplasias malignas da segunda edição da Classificação Internacional do Câncer na Infância (CICI) e códigos C00 a C97 (Capítulo II - Neoplasias) da Classificação Estatística Internacional de Doenças e Problemas Relacionados à Saúde (CID-10), em menores de 20 anos de idade, residentes em cada município no período de 2000 a 2005, classificação própria do RCBP de Mato Grosso - criado em 1999 e a coleta de dados iniciada no ano de 2000 em Cuiabá e Várzea Grande, sendo implementada aos municípios do interior do estado a partir de 2001. Encontra-se sob a coordenação do serviço de vigilância epidemiológica da Secretaria de Estado da Saúde de Mato Grosso, e recebe suporte financeiro fixo da Secretaria de Vigilância em Saúde do Ministério da Saúde (SVS/MS), conforme regulamentado pela Portaria no $2.607 / \mathrm{GM}$ de $28 \mathrm{de}$ dezembro de 2005.

\section{Mortalidade por câncer}

Óbitos por neoplasias malignas - códigos $\mathrm{C} 00$ a C97 (Capítulo II - Neoplasias) da CID 10 - em menores de 20 anos de idade, residentes em cada município no período de 2000 a 2006, utilizando-se as informações dos bancos de dados de registros vitais do Ministério da Saúde, através do Sistema de Informações sobre Mortalidade (SIM) do Departamento de Informática do Sistema Único de Saúde ${ }^{16}$.

\section{Uso de agrotóxicos (proxi da exposição)}

O banco de dados sobre o uso de agrotóxicos no estado no período de 2005 a 2009 foi obtido junto ao Instituto de Defesa Agropecuária do Estado de Mato Grosso (INDEA-MT) ${ }^{17} \mathrm{e}$ disponibilizado por Pignati. Para o consumo de agrotóxicos dos anos de 2000 a 2004, utilizou-se como unidade de correção o volume de agrotóxico por hectare de cada tipo de lavoura temporária nos anos de 2005 a 2009 em cada município do estado e aplicou-se essa média sobre as áreas de lavoura temporária dos anos anteriores ${ }^{14}$.
No estudo ecológico, as limitações estão descritas na literatura como: falácia ecológica, quando não é possível associar exposição e doença no nível individual; dificuldade de controlar os efeitos de potenciais fatores de confundimento; os dados de estudos ecológicos representam níveis de exposição média em vez de valores individuais reais; falta de disponibilidade de informações relevantes, dificuldade de separar estatisticamente efeitos específicos de certas variáveis (colinearidade).

\section{RESULTADOS}

\section{O uso de agrotóxicos em Mato Grosso}

A utilização dos agrotóxicos acompanha o volume e a diversidade da produção agrícola. No estado de Mato Grosso, as culturas temporárias como os cultivos extensivos de soja, algodão e milho, demandam intenso uso de agrotóxicos ${ }^{14,18}$.

Como a maioria da produção agrícola é dependente de alta tecnologia e de agroquímicos, utilizaram-se no estado, no ano de 2005, aproximadamente 75 mil toneladas de agrotóxicos (formulações comerciais), calculado pelo consumo médio de agrotóxicos por hectare de lavoura temporária, segundo o INDEA-MT ${ }^{17}$.

O estado de Mato Grosso tornou-se o maior consumidor nacional destes produtos tóxicos (inseticida, herbicida, fungicida e outros) perfazendo $19 \%$ de todo o consumo brasileiro ${ }^{19}$.

Dessa forma, a evolução da utilização de agrotóxicos no estado apresentou incremento de $89,10 \%$ no período entre 2000 e 2005, considerando somente a área de lavoura temporária.

As informações acerca da venda de agrotóxicos específicos por classe e ingredientes químicos se tornaram disponíveis a partir de $2005 \mathrm{em}$ um relatório produzido pelo INDEA-MT, permitindo a realização de análise dos mais utilizados no estado.

A média anual de agrotóxicos comercializados em Mato Grosso nos anos de 2005 a 2009 foi de aproximadamente 80 milhões de litros, sendo $48 \%$ de herbicidas, 33\% de inseticidas, $13 \%$ de fungicidas e $6 \%$ dos ingredientes ativos não foram especificados (Figura 1).

Dos agrotóxicos comercializados no estado entre os anos de 2005 a 2009, pelo menos, 48,91\% foram apontados nos estudos como carcinogênicos. Dentre os mais usados estão o glifosato (25,91\%), o endossulfan (6,47\%), o $2,4 \mathrm{D}(5,58 \%)$ e o tebuconazol $(5,14 \%)$.

Dos agrotóxicos mais utilizados no estado, $23,64 \%$ são apontados como possivelmente carcinogênicos, isto é, são produtos que podem ser metabolizados em carcinógenos efetivos. 
Dentre eles salientam-se o metamidofós (7,70\%), a atrazina $(3,86 \%)$, o carbendazim $(1,91 \%)$ e a clomazona (1,33\%). Dos agrotóxicos comercializados no estado entre os anos de 2005 e 2009 , apenas $11,95 \%$ não são comprovadamente carcinogênicos.

Entretanto, 7,08\% destes eram mutagênicos e é importante esclarecer que determinadas mutações somáticas - aquelas que se propagam pelo corpo do indivíduo - estão relacionadas à indução de processos carcinogênicos ${ }^{18,20}$.

\section{Incidência do câncer infantojuvenil em Mato Grosso}

O estado de Mato Grosso possui uma população jovem. No censo do ano de $2010,35,05 \%$ da população mato-grossense encontrava-se abaixo dos 20 anos de idade ${ }^{21}$.

Nas informações do RCBP de Mato Grosso para o período de 2000 a 2005 foram registrados 702 pacientes com câncer com idade entre 0 e 19 anos, representando 3,26\% do total de casos de câncer admitidos, sendo 377 do sexo masculino e 325 do feminino. As crianças (menores de 15 anos) representaram $2,31 \%$ do total de casos admitidos e os adolescentes (entre 15 e 19 anos) representaram 0,94\% do total.

No período de estudo, o RCBP-MT registrou que, entre as crianças, ocorreram 176 casos de leucemias (35,27 \%), 77 de linfomas (15,43\%) e 70 de tumores do SNC e miscelâneas de neoplasias intracranianas e intraespinais (14,03\%). Entre os adolescentes, ocorreram 48 casos de carcinomas e outras neoplasias malignas epiteliais (23,65\%), 38 leucemias (18,72\%), 29 linfomas (14,29\%) e 28 tumores ósseos malignos $(13,79 \%)$.
A Figura 2 apresenta a distribuição das neoplasias mais frequentes para as faixas etárias entre 0 e 19 anos.

Na Figura 2, observou-se no período estudado que a incidência de leucemias foi de 30,48\% (214 casos) para menores de 20 anos, sendo $35,27 \%$ em menores de 15 anos e $18,72 \%$ nos adolescentes. O grupo de linfomas e neoplasias retículo-endoteliais corresponde ao segundo mais incidente $(15,10 \%)$ no período, sendo a maioria linfoma não Hodgkin, no sexo masculino e em menores de 15 anos.

Observou-se ainda, na Figura 2, que o terceiro grupo mais incidente foi de tumores de SNC e miscelânia de neoplasias intracranianas e intraespinais (70 casos em crianças e 17 em adolescentes), sendo neoplasias intracranianas e intraespinais não especificadas (44 casos), astrocitomas (20 casos) e tumores neuroectodérmicos primitivos (13 casos).

Registraram-se para os demais tipos de câncer infantojuvenil, no período de estudo, 20 casos (4\%) no grupo Sistema Nervoso Simpático em menores de 15 anos; 1,6\% de retinoblastoma entre os menores de 15 anos (todos os casos registrados até o sexto ano de vida); 27 casos de tumores renais em menores de 15 anos, correspondendo a 5,41\% dos tumores infantis; 3 casos de hepatoblastoma em menores de 1 ano de idade e 1 hepatocarcinoma em adolescente; 7,55\% de tumores ósseos malignos, sendo 4,27\% de osteossarcomas na faixa etária entre $10-19$ anos; 4,99\% de casos no grupo dos sarcomas de partes moles; 3,28\% de neoplasias de células germinativas, trofoblásticas e outras gonadais e $9,97 \%$ de carcinomas e outras neoplasias epiteliais.

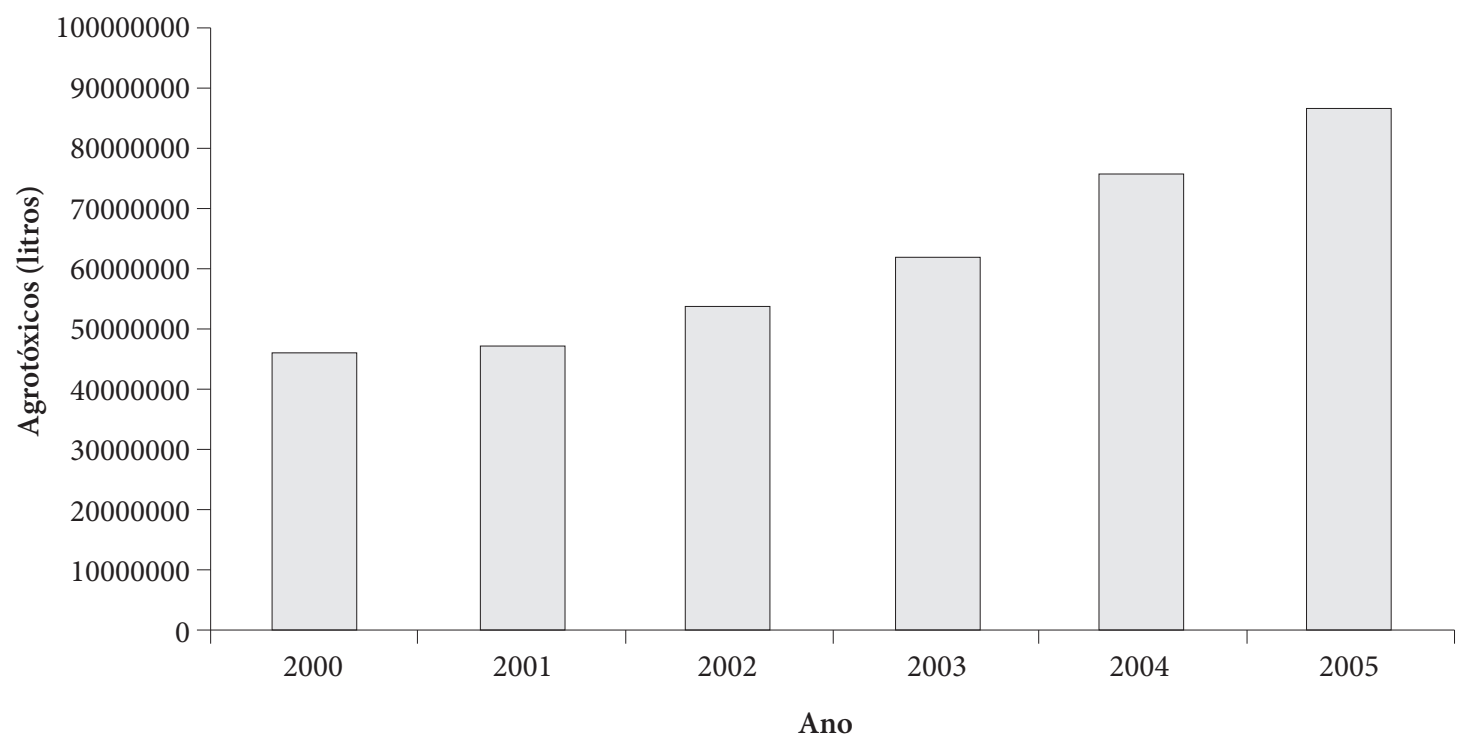

Fonte: Pignati ${ }^{14}$, IBGE ${ }^{21}$ — organizado pela autora

Figura 1. Evolução da utilização de agrotóxicos em Mato Grosso, em litros, no período de 2000 a 2005 
No RCBP-MT, em um estudo da procedência segundo municípios de residência dos pacientes menores de 20 anos diagnosticados no período de 2000-2005 observou-se que $42,74 \%$ provêm da região metropolitana de Cuiabá e Várzea Grande e 57,26\% dos municípios do interior do estado.

\section{Mortalidade infantojuvenil por câncer em Mato Grosso}

A mortalidade por câncer em crianças e adolescentes com idade entre 0 e 19 anos passou de 2,97\%, em 2000, para 3,76\%, em 2006, dos óbitos registrados no estado de Mato Grosso. No ano de 2006, configurou-se como a segunda causa de morte nesta faixa etária, correspondendo a $8 \%$ de todos os óbitos (9,52\% entre 0 e 14 anos de idade).

Na Tabela 1, distribuíram-se os tipos de óbitos por câncer em menores de 20 anos, segundo sexo nos anos de 2000 a 2006, no estado de Mato Grosso.

Observou-se que, de todos os óbitos por câncer em menores de 20 anos registrados no SIM/DATASUS entre os anos de 2000 e 2006, no estado de Mato Grosso, as leucemias representaram a maior causa, sendo responsáveis por 31,94\%; os tumores do SNC foram a segunda causa dos óbitos (20,16\%); os linfomas não Hodgkin foram responsáveis por $7,07 \%$ e os tumores hepáticos por $2 \%$ dos óbitos.

Associação entre câncer infantojuvenil e uso de agrotóxicos nos municípios de Mato Grosso

Para analisar a associação entre o uso de agrotóxicos nos municípios do estado e o câncer em crianças e adolescentes, optou-se por trabalhar com as médias, tendo em vista diferentes fontes, períodos e unidades de mensuração dos dados disponíveis. Sendo que dos 141 municípios existentes, 88 apresentaram casos novos e 85 tiveram óbitos nesta faixa etária, nos períodos estudados, conforme Tabela 2 .

Dessa forma, foi testada a correlação entre a média de uso de agrotóxicos, de casos novos e de óbitos por câncer, na faixa etária de 0-19 anos, nos municípios de Mato Grosso, nos períodos apresentados.

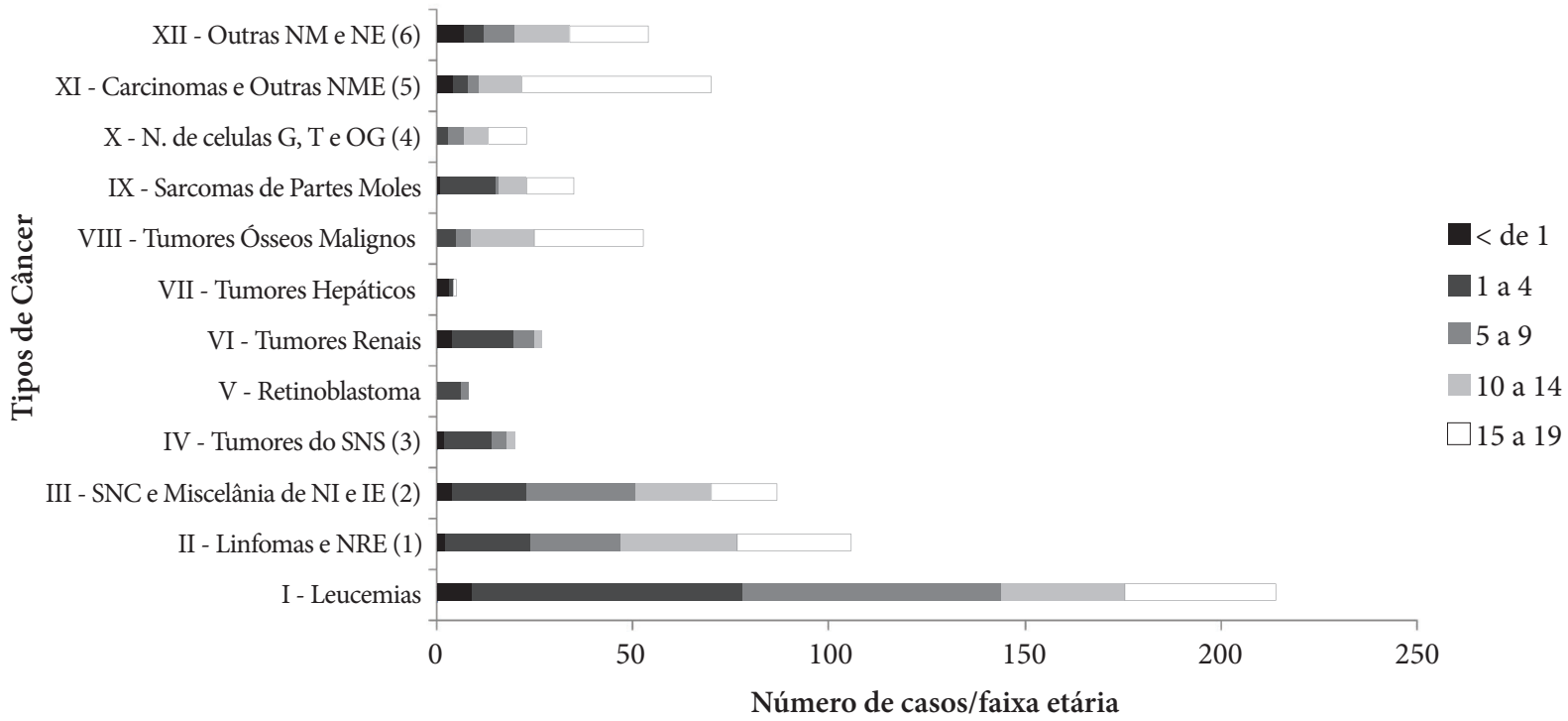

Nota: (1) Neoplasias retículo endoteliais; (2) Neoplasias intracranianas e intra-espinhais; (3) Sistema nervoso simpático; (4) Células germinativas, trofoblásticas e outras gonadais; (5) Neoplasias malignas epiteliais e (6) Neoplasias malignas e não especificadas.

Fonte: INCA-RCBPMT29

Figura 2. Número de casos por tipo de câncer infantojuvenil, segundo faixa etária — Mato Grosso (2000-2005)

Tabela 1. Distribuição de óbitos e percentual de mortalidade por sexo, segundo causa básica, em menores de 20 anos — Mato Grosso (2000-2006)

\begin{tabular}{|c|c|c|c|c|c|c|}
\hline \multirow{2}{*}{ Causa básica } & \multicolumn{2}{|c|}{ Masculino } & \multicolumn{2}{|c|}{ Feminino } & \multicolumn{2}{|c|}{ Total } \\
\hline & $\mathbf{n}$ & $\%$ & $\mathbf{n}$ & $\%$ & $\mathbf{n}$ & $\%$ \\
\hline Leucemias & 70 & 33,33 & 52 & 30,23 & 122 & 31,94 \\
\hline Sistema Nervoso Central & 40 & 19,05 & 37 & 21,51 & 77 & 20,16 \\
\hline Linfomas & 22 & 10,48 & 5 & 2,91 & 27 & 7,07 \\
\hline Outras neoplasias & 78 & 37,14 & 78 & 45,35 & 156 & 40,84 \\
\hline Total & 210 & 54,97 & 172 & 45,03 & 382 & 100,00 \\
\hline
\end{tabular}

Fonte: SIM/DATASUS/MS ${ }^{16}$ 
Tabela 2. Relação dos municípios, média de uso de agrotóxicos e a morbidade (2001-2005) e a mortalidade (2000-2006) por câncer em menores de 20 anos - Mato Grosso

\begin{tabular}{|c|c|c|c|c|c|c|c|c|c|}
\hline 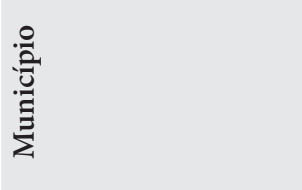 & 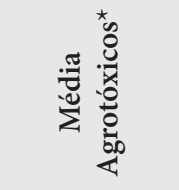 & 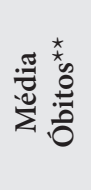 & 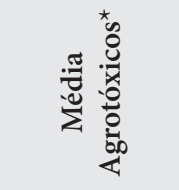 & 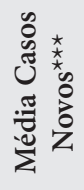 & $\frac{\stackrel{ }{\frac{3}{3}}}{\stackrel{\Xi}{\Xi}}$ & 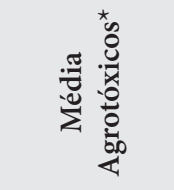 & 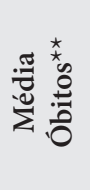 & 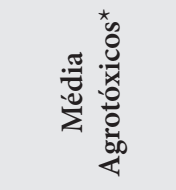 & 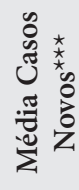 \\
\hline Acorizal & $1.091,36$ & 0,14 & $1.105,91$ & 0,60 & Nobres & $17.976,64$ & 0,86 & $17.788,39$ & 0,60 \\
\hline Água Boa & $287.425,00$ & 0,29 & $305.797,88$ & 0,40 & Nortelândia & $15.3367,20$ & 0,14 & $157.526,23$ & 0,40 \\
\hline Alta Floresta & $101.802,00$ & 0,71 & $102.887,53$ & 1,60 & Nossa Sra. Livramento & $1.975,16$ & 0,14 & & 0,00 \\
\hline Alto Araguaia & & 0,00 & $90.509,84$ & 0,40 & Nova Bandeirantes & $11.593,24$ & 0,14 & $9.052,15$ & 0,40 \\
\hline Alto Boa Vista & $26.254,61$ & 0,14 & & 0,00 & Nova Brasilândia & & 0,00 & $44.141,28$ & 0,40 \\
\hline Alto Paraguai & $31.842,39$ & 0,43 & $30.873,82$ & 0,60 & Nova Canaã do Norte & $88.395,97$ & 0,14 & $83.730,56$ & 0,20 \\
\hline Alto Taquari & $452.475,20$ & 0,14 & $462.418,68$ & 0,40 & Nova Lacerda & & 0,00 & $63.953,07$ & 0,20 \\
\hline Araputanga & $26.148,91$ & 0,43 & $25.998,91$ & 1,20 & Nova Marilândia & & 0,00 & $23.075,02$ & 0,40 \\
\hline Arenápolis & $11.868,07$ & 0,29 & $14.598,56$ & 0,60 & Nova Maringá & & 0,00 & $204.669,45$ & 0,20 \\
\hline Aripuanã & $9.996,86$ & 0,43 & $7.656,25$ & 0,80 & Nova Monte Verde & $57.726,16$ & 0,14 & & 0,00 \\
\hline Barão de Melgaço & & 0,00 & $1.148,80$ & 0,40 & Nova Mutum & & 0,00 & $2.625 .668,03$ & 1,20 \\
\hline Barra do Bugres & $143.316,00$ & 0,86 & $148.738,16$ & 2,60 & Nova Olímpia & $119.150,80$ & 0,29 & $120.051,91$ & 0,40 \\
\hline Barra do Garças & $167.315,90$ & 0,71 & $160.613,38$ & 0,80 & Nova Ubiratã & $875.690,20$ & 0,14 & & 0,00 \\
\hline Brasnorte & $1381.220,00$ & 0,29 & $1.405 .823,05$ & 1,00 & Nova Xavantina & $513.864,00$ & 0,57 & $556.114,37$ & 1,40 \\
\hline Cáceres & $85.348,22$ & 1,14 & $84.019,31$ & 3,60 & Novo Horizonte Norte & & 0,00 & $5.207,79$ & 0,20 \\
\hline Campinápolis & $10.892,07$ & 0,14 & & 0,00 & Novo São Joaquim & $770.908,00$ & 0,14 & $673.818,87$ & 0,20 \\
\hline Campo Novo do Parecis & $7.178 .558,00$ & 0,71 & $7.046 .206,20$ & 0,80 & Paranaíta & $141.201,80$ & 0,29 & $134.029,47$ & 0,60 \\
\hline Campo Verde & $3.344 .861,00$ & 0,71 & $3.387 .420,34$ & 1,40 & Paranatinga & & 0,00 & $154.825,72$ & 0,80 \\
\hline Canabrava do Norte & $13.744,21$ & 0,29 & $14.475,71$ & 0,20 & Peixoto de Azevedo & $4.639,68$ & 0,86 & $4.445,31$ & 0,60 \\
\hline Canarana & $733.663,10$ & 0,43 & $771.154,61$ & 0,40 & Poconé & $1.467,67$ & 0,57 & $1.527,54$ & 2,00 \\
\hline Carlinda & $55.865,45$ & 0,14 & $43.784,30$ & 0,20 & Ponte Branca & 499,43 & 0,14 & & 0,00 \\
\hline Castanheira & $17.564,50$ & 0,14 & $18.565,36$ & 0,20 & Pontes e Lacerda & $36.029,88$ & 1,14 & $32.518,25$ & 1,80 \\
\hline Chapada dos Guimarães & $51.526,03$ & 0,57 & $47.837,88$ & 0,60 & Porto Alegre do Norte & $11.801,48$ & 0,14 & & 0,00 \\
\hline Cláudia & $53.342,81$ & 0,14 & $57.498,67$ & 1,00 & Porto dos Gaúchos & $95.054,62$ & 0,29 & $105.899,67$ & 0,80 \\
\hline Colíder & $36.596,14$ & 0,57 & $32.157,74$ & 2,20 & Porto Esperidião & $17.531,64$ & 0,14 & $19.055,59$ & 0,40 \\
\hline Colniza & $1.843,12$ & 0,29 & & 0,00 & Poxoréo & $620.612,20$ & 0,43 & $636.059,54$ & 0,80 \\
\hline Comodoro & $212.119,10$ & 0,43 & $192.616,12$ & 0,40 & Primavera do Leste & $5.619 .557,00$ & 0,71 & $5.858 .704,84$ & 2,00 \\
\hline Conquista D'Oeste & & 0,00 & $8.005,04$ & 0,20 & Querência & $906.549,30$ & 0,14 & $863.312,64$ & 0,20 \\
\hline Cuiabá & $8.418,83$ & 12,29 & $8.298,09$ & 31,80 & Reserva do Cabaçal & $1.177,60$ & 0,29 & & 0,00 \\
\hline Curvelândia & $1.837,66$ & 0,14 & $2.075,90$ & 0,20 & Ribeirãozinho & $5.209,40$ & 0,14 & & 0,00 \\
\hline Denise & $1.584,18$ & 0,14 & $1.663,60$ & 1,00 & Rondonópolis & $1.111 .729,00$ & 3,57 & $1.095 .792,39$ & 10,80 \\
\hline Diamantino & $2.693 .253,00$ & 0,29 & $2.722 .849,02$ & 0,40 & Rosário Oeste & $15.216,14$ & 0,29 & $16.128,29$ & 0,80 \\
\hline Dom Aquino & $304.088,70$ & 0,14 & $309.721,52$ & 0,40 & Salto do Céu & & 0,00 & $7.334,91$ & 0,20 \\
\hline Feliz Natal & $23.619,80$ & 0,43 & $22.465,53$ & 0,20 & Santa Terezinha & 48022,30 & 0,14 & & 0,00 \\
\hline Gaúcha do Norte & $96.107,46$ & 0,14 & & 0,00 & Santo Afonso & & 0,00 & $2.756,20$ & 0,20 \\
\hline Glória D’Oeste & $6.475,33$ & 0,14 & & 0,00 & Santo Antônio Leverger & $44.679,14$ & 0,14 & $49.043,13$ & 1,20 \\
\hline Guarantã do Norte & $13.195,58$ & 0,43 & $11.429,49$ & 0,80 & São Félix do Araguaia & $13.252,47$ & 0,14 & & 0,00 \\
\hline Guiratinga & $432.610,00$ & 0,71 & $419.234,73$ & 1,20 & São José do Povo & & 0,00 & $3.409,64$ & 0,60 \\
\hline Indiavaí & $2.646,68$ & 0,14 & $2.762,70$ & 0,20 & São José do Rio Claro & $297.182,10$ & 0,14 & & 0,00 \\
\hline Itaúba & $87.976,65$ & 0,43 & $92.499,72$ & 0,40 & São José Quatro Marcos & $55.180,74$ & 0,71 & $56.153,30$ & 1,00 \\
\hline Jaciara & $384.808,30$ & 0,43 & $382.536,05$ & 0,80 & Sinop & $992.245,10$ & 1,43 & $1.019 .130,58$ & 4,80 \\
\hline Jangada & $6.917,67$ & 0,57 & $7.284,49$ & 0,80 & Sorriso & $3.735 .864,00$ & 1,29 & $3.755 .971,42$ & 2,60 \\
\hline Jauru & $2.035,20$ & 0,14 & $2.090,98$ & 0,40 & Tangará da Serra & $1.044 .001,00$ & 1,00 & $1.052 .614,67$ & 2,80 \\
\hline Juara & $47.961,63$ & 0,71 & $49.907,20$ & 1,00 & Tapurah & $1.144 .353,00$ & 0,29 & $1.318 .943,26$ & 0,60 \\
\hline Juína & $37.554,40$ & 1,00 & $38.708,48$ & 1,80 & Terra Nova do Norte & $6.562,32$ & 0,43 & $6.629,53$ & 1,00 \\
\hline Juruena & & 0,00 & $11.466,34$ & 0,20 & Tesouro & & 0,00 & $116.655,91$ & 0,20 \\
\hline Juscimeira & $155.379,20$ & 0,43 & $153.980,99$ & 0,20 & União do Sul & $13.562,91$ & 0,14 & $14.015,41$ & 1,20 \\
\hline Lambari D’Oeste & & 0,00 & $29.628,62$ & 0,20 & Vale de São Domingos & 329,27 & 0,14 & 0,00 & 0,00 \\
\hline Lucas do Rio Verde & $3.176 .834,00$ & 0,43 & $3.209 .992,16$ & 1,00 & Várzea Grande & $5.146,26$ & 6,00 & $4.890,23$ & 15,40 \\
\hline Luciara & & 0,00 & 193,61 & 0,60 & Vera & & 0,00 & $189.888,47$ & 0,40 \\
\hline Marcelândia & $7.630,62$ & 0,14 & $7.778,39$ & 0,60 & Vila Bela Sma. Trindade & & 0,00 & $44.677,95$ & 0,20 \\
\hline Mirassol d'Oeste & $15.724,19$ & 0,29 & $16.554,71$ & 0,60 & Vila Rica & 281,63 & 0,29 & 271,54 & 0,40 \\
\hline
\end{tabular}

Fonte: *Pignati ${ }^{14 ; *}$ SIM/DATASUS/MS ${ }^{16 ; * * *}$ INCA-RCBPMT ${ }^{29}$ 
Tabela 3. Associação entre média de uso de agrotóxicos nos municípios de Mato Grosso e casos novos e óbitos por câncer infantojuvenil nos períodos apresentados

\begin{tabular}{|c|c|c|c|}
\hline Correlação & $\begin{array}{c}\text { Média de } \\
\text { agrotóxicos }^{\star * *}\end{array}$ & $\begin{array}{l}\text { Número de } \\
\text { municípios }\end{array}$ & Período \\
\hline $\begin{array}{l}\text { Média de casos } \\
\text { novos de câncer IJ }\end{array}$ & $\mathrm{p}=0,021$ & 85 & 2001-2005 \\
\hline $\begin{array}{l}\text { Média de óbitos } \\
\text { por câncer IJ }{ }^{\star *}\end{array}$ & $\mathrm{p}=0,005$ & 82 & 2000-2006 \\
\hline
\end{tabular}

IJ: infantojuvenil.

Fonte: *INCA-RCBPMT ${ }^{29 ;}$; ${ }^{*}$ SIM/DATASUS/MT ${ }^{16}$; ***Pignati ${ }^{14}$

Na Tabela 3, observou-se que a média de uso de agrotóxicos nos munícipios apresentou associação estatisticamente significante tanto para morbidade por câncer em menores de 20 anos $(\mathrm{p}=0,021)$, como para mortalidade por câncer infantojuvenil $(\mathrm{p}=0,005)$, com intervalo de confiança de $95 \%$.

Cabe salientar que foram excluídos os municípios de Cuiabá, Várzea Grande e Rondonópolis a fim de caracterizar o uso agrícola de agrotóxicos, pois estes municípios são polos industriais, comerciais e conglomerados urbanos.

\section{DISCUSSÃO}

O intenso uso de agrotóxicos no estado de Mato Grosso configura-se em um dos fatores ambientais de grande relevância para explicar o aumento de alguns tipos de câncer na faixa etária de 0-19 anos. A maior parte do uso de agrotóxicos no estado está relacionada à agricultura.

Cunha ${ }^{22}$ registrou a distribuição da mortalidade por câncer e os padrões de consumo de agrotóxicos nas regiões do estado de Mato Grosso no período de 1998-2006.

Por outro lado, durante os anos de 2007 a $2010 \mathrm{em}$ pesquisa realizada no município de Lucas do Rio Verde, Moreira et al. ${ }^{15}$ observaram exposição ambiental/ocupacional/alimentar de 136 litros de agrotóxicos por habitante, contaminação com resíduos de agrotóxicos em poços de água potável, amostras de chuva, amostras de ar, amostras de sangue e urina de professores; Dos Santos et al. ${ }^{23}$ validaram método analítico para determinação de agrotóxicos no ambiente e detectaram resíduos no ar do município; Palma ${ }^{24}$ detectou resíduos de agrotóxicos em leite materno; Fávero ${ }^{25}$ avaliou agravos respiratórios em menores de cinco anos associados às pulverizações de agrotóxicos em lavouras do mesmo município.

Pignati e Machado $^{26}$ avaliaram o impacto da poluição intencional por agrotóxicos, especialmente na população do interior através dos agravos à saúde e dos danos ambientais.

Para o Brasil, o estudo de Ribeiro et al. ${ }^{27}$ demonstrou uma tendência decrescente na mortalidade por leucemias para o período entre 1980 e 2002. Segundo o autor, essa redução foi maior nos estados mais desenvolvidos. Porém, isso não ocorreu em Mato Grosso no período estudado, pois a tendência linear foi de crescimento.

Os linfomas foram o segundo grupo de tumores mais incidentes e apresentaram tendência decrescente na mortalidade no grupo infantojuvenil, no período estudado, em Mato Grosso.

As taxas de mortalidade para tumores cerebrais no Brasil foram analisadas por Monteiro e Koifman ${ }^{28}$, em 2003, quando observaram um crescimento de 2,24/100.000 habitantes para 3,35/100.000 habitantes, correspondendo a um aumento de 50\% no período estudado (1980-1998). Essas taxas foram mais elevadas na infância que na adolescência, aumentando posteriormente com a idade. Os autores sugerem que este crescimento pode ser explicado parcialmente pela maior disponibilidade de acesso aos cuidados com a saúde, em particular aos meios de diagnóstico por imagem. Em Mato Grosso, as taxas de mortalidade por tumores do SNC mantiveram-se estáveis no período estudado.

\section{CONCLUSÃO}

Os achados neste estudo indicam que a exposição desde o nascimento às transformações ocasionadas pelo modelo produtivo, especialmente aos agrotóxicos, tem relação estatisticamente significante com os indicadores de morbidade e de mortalidade por câncer em menores de 20 anos nos municípios do estado de Mato Grosso, nos períodos estudados.

Recomendam-se como medidas de promoção da saúde e prevenção do câncer: estabelecer um sistema intersetorial de vigilância do uso agrícola de agrotóxicos e notificação de casos de câncer infantojuvenil; monitorar a notificação de casos de câncer infantojuvenil em municípios com intensa atividade agrícola e fiscalizar a utilização de agrotóxicos potencial e comprovadamente cancerígenos estabelecendo ações para a proibição do uso e minimização de risco no nível municipal.

As ações para o diagnóstico precoce da doença devem ser desenvolvidas prioritariamente nestas áreas através da atuação efetiva da Atenção Básica no acompanhamento, vigilância e promoção da saúde da criança e do adolescente; de estratégias de divulgação de informações para profissionais e para a população, ressaltando a importância do diagnóstico precoce e o aumento da comunicação entre os serviços de cuidado primário e os especializados para tratamento. 


\section{REFERÊNCIAS}

1. Brasil. Ministério da Saúde. Instituto Nacional de Câncer (INCA). Particularidades do câncer infantil. [cited 2011 Mar 20]. Available from: http://www.inca.gov.br/conteudo_view.asp?id=343

2. Zahm SH, Ward MH. Pesticides and childhood cancer. Environ Health Perspect. 1998;106(Suppl 3):893-908.

3. Brasil. Ministério da Saúde. Instituto Nacional de Câncer (INCA). Diagnóstico precoce do câncer na criança e no adolescente. Rio de Janeiro: Instituto Nacional do Câncer; 2009.

4. Brasil. Ministério da Saúde. Instituto Nacional de Câncer (INCA). Coordenação de Prevenção e Vigilância de Câncer. Câncer da criança e adolescente no Brasil: dados dos registros de base populacional e de mortalidade. Rio de Janeiro: Instituto Nacional do Câncer; 2008.

5. Braga PE, Latorre MRD, Curado MP. Câncer na infância: análise comparativa da incidência, mortalidade e sobrevida em Goiânia (Brasil) e outros países. Cad Saúde Pública. 2002;18(1):33-44.

6. Brasil. Ministério da Saúde. Agência Nacional de Vigilância Sanitária. Vigilância do câncer ocupacional e ambiental. Rio de Janeiro: Instituto Nacional do Câncer; 2006.

7. Martos CM, Olsen JH. Childhood cancer mortality in the European Community, 1950-1989. Eur J Cancer. 1993;29A(12):1783-9.

8. Levi F, La Vecchia C, Lucchini F, Negri E, Boyle P. Patterns of childhood cancer mortality: America, Asia and Oceania. Eur J Cancer.1995;31A(5):771-82.

9. Little J. Introduction. In: Little J. Epidemiology of childhood cancer. Lyon: International Agency for Research on Cancer: World Health Organization; 1999. p. 1-9. [IARC Scientific Publications, 149].

10. Ries LAG, Smith MA, Gurney JG, Linet M, Tamra T, Young JL, et al. editors. Cancer incidence and survival among children and adolescents: United States SEER Program 1975-1995. Bethesda: National Cancer Institute; 1999 [cited 2008 Oct 2]. Available from: http://seer.cancer.gov/ publications/childhood/

11. Nunes MV, Tajara EH. Efeitos tardios dos praguicidas organoclorados no homem. Rev Saúde Pública.1998;32(4):372-83.

12. Miligi L, Costantini AS, Veraldi A, Benvenuti A; Will, Vineis P. Cancer and pesticides: an overview and some results of the Italian multicenter case-control study on hematolymphopoietic malignancies. Ann NY Acad Sci. 2006;1076:366-77

13. Sanborn M, Kerr KJ, Sanin LH, Cole DC, Bassil KL, Vakil C. Non-cancer Health effects of pesticides: systematic review and implications for family doctors. Can Fam Physician. 2007;53(10):1712-20.

14. Pignati WA. Os riscos, agravos e vigilância em saúde no espaço de desenvolvimento do agronegócio no Mato Grosso [tese]. Rio de Janeiro (RJ): Fundação Oswaldo Cruz. Escola Nacional de Saúde Pública 2007.

15. Moreira JC, Peres F, Pignati WA, Dores EFGC. Avaliação do risco à saúde humana decorrente do uso de agrotóxicos na agricultura e pecuária na região Centro oeste. Relatório de pesquisa. Brasília; 2010. CNPq 555193/2006-3.
16. Brasil. Ministério da Saúde. Informações de Saúde. [cited 2011Dec 11]. Available from: http://tabnet.datasus.gov.br/cgi/deftohtm.exe?sim/cnv/ inf10mt.def

17. Instituto de Defesa Agropecuária de Mato Grosso (INDEA). Relatório de consumo de agrotóxico em Mato Grosso. Banco eletrônico. Cuiabá; 2010.

18. Grisólia KC. Agrotóxicos-mutações, reprodução e câncer. Brasília: Editora Universidade de Brasília, 2005.

19. Sindicato das Indústrias de Defensivos Agrícolas (SINDAG). Seminário Nacional Sobre Agrotóxicos, Saúde e Ambiente. Agência Nacional de Vigilância Sanitária, Ministério Público Federal e Secretaria de Estado de Saúde de Pernambuco; 2005 Oct; Olinda, Pernambuco.

20. Zakrzewski, SF. Principles of environmental toxicology. American Chemical Society. 1991;270.

21. Instituto Brasileiro de Geografia e Estatística (IBGE). Censo Demográfico 2010. [cited 2011 Mar 20]. Available from: http://www.ibge.gov.br/home/ mapa_site/mapa_site.php\#indicadores

22. Cunha MLON. Mortalidade por câncer e a utilização de pesticidas no estado de Mato Grosso [dissertação]. São Paulo (SP); Faculdade de Medicina da Santa Casa de São Paulo; 2010.

23. Dos Santos LG, Lourencetti C, Pinto AA, Pignati WA, Dores EF Validation and application of an analytical method for determining pesticides in the gas phase of ambient air. J Environ Sci Health B. 2011;46(2):150-62.

24. Palma DCA. Agrotóxicos em leite humano de mães residentes em Lucas do Rio Verde-Mato Grosso [dissertação]. Cuiabá (MT): Universidade Federal de Mato Grosso; 2011.

25. Fávero KAS. Pulverizações de agrotóxicos nas lavouras de Lucas do Rio Verde e os agravos respiratórios em crianças menores de 5 anos [dissertação]. Cuiabá (MT): Universidade Federal de Mato Grosso; 2011.

26. Pignati WA, Machado JMH. O agronegócio e seus impactos na saúde dos trabalhadores e da população do Estado de Mato Grosso. In: Gomez CM, Machado JMH, Pena PGL, organizadores. Saúde do trabalhador na sociedade brasileira contemporânea. Rio de Janeiro: Editora Fundação Oswaldo Cruz; 2011. p. 245-72.

27. Ribeiro KCB, Lopes LF, de Camargo B. Trends in childhood leukemia mortality in Brazil and correlation with social inequalities. Cancer. 2007;110(8):1823-31.

28. Monteiro GTR, Koifman S. Mortalidade por tumores de cérebro no Brasil, 1980-1998. Cad Saúde Pública. 2003;19(4):1139-51.

29. Brasil. Ministério da Saúde. Instituto Nacional de Câncer (INCA) Coordenação de Prevenção e Vigilância de Câncer. [cited 2011 Dec 11]. Available from: http://www2.inca.gov.br/wps/wcm/connect/estatisticas/ site/home/rcbp/

Recebido em: 25/04/2012 Aprovado em: 19/10/2012 